Claremont Colleges

Scholarship@ Claremont

All HMC Faculty Publications and Research

HMC Faculty Scholarship

$1-1-1987$

\title{
Sorting and Selecting in Rounds
}

Nicholas Pippenger

Harvey Mudd College

\section{Recommended Citation}

Nicholas Pippenger. "Sorting and Selecting in Rounds", Society for Industrial and Applied Mathematics Journal on Computing, 16, 1032 (1987).

This Article is brought to you for free and open access by the HMC Faculty Scholarship at Scholarship @ Claremont. It has been accepted for inclusion in All HMC Faculty Publications and Research by an authorized administrator of Scholarship @ Claremont. For more information, please contact scholarship@cuc.claremont.edu. 


\title{
SORTING AND SELECTING IN ROUNDS*
}

\author{
NICHOLAS PIPPENGER $\dagger$
}

\begin{abstract}
We present upper bounds for sorting and selecting the median in a fixed number of rounds. These bounds match the known lower bounds to within logarithmic factors. They also have the merit of being "explicit modulo expansion"; that is, probabilistic arguments are used only to obtain expanding graphs, and when explicit constructions for such graphs are found, explicit algorithms for sorting and selecting will follow. Using the best currently available explicit constructions for expanding graphs, we present the best currently known explicit algorithms for sorting and selecting in rounds.
\end{abstract}

Key words. sorting, selecting, median, parallel computation

AMS(MOS) subject classification. $68 \mathrm{P} 10$

1. Introduction and history. The three principal problems of comparison-treebased complexity theory are sorting, merging and selecting an element of prescribed rank (such as the minimum, maximum or median). For each problem we may study the worst-case complexity using Valiant's parallel comparison-tree model [V]. There are three commonly studied degrees of parallelism for these problems: the case of a single "processor" (or a fixed number of processors), which we shall call the serial case; the case of a number of processors equal to the number of elements, which we shall call the balanced case; and the case of a number of processors large enough to allow the solution to be found in a fixed number of "rounds," which we shall call the highly parallel case.

For sorting $n$ elements it has long been known that, in the serial case, $\Theta(n \log n)$ steps are needed. This implies that $\Omega(\log n)$ steps are needed in the balanced case; Ajtai, Komlós and Szemerédi [AKS] showed that $O(\log n)$ steps are sufficient. For the highly parallel case, Häggkvist and Hell [HH2] showed that $\Omega\left(n^{1+1 / k}\right)$ comparisons are needed to sort in $k$ rounds; this has been improved to $\Omega\left(n^{1+1 / k}(\log n)^{1 / k}\right)$ by Alon, Azar and Vishkin [AAV]. Bollobás and Thomason [BT] showed that $O\left(n^{3 / 2} \log n\right)$ comparisons are sufficient to sort in 2 rounds. This has been improved by Alon, Azar and Vishkin [AAV] to $O\left(n^{3 / 2} \log n /(\log \log n)^{1 / 2}\right)$ comparisons, and generalized by Bollobás and Hell [BH1] to $O\left(n^{1+1 / k} \log n\right)$ comparisons for $k$ rounds. In this paper we shall show that $O\left(n^{1+1 / k}(\log n)^{2-2 / k}\right)$ comparisons are sufficient to sort in $k$ rounds. While slightly larger than the upper bound reported by Bollobás and Hell, this result has the merit of being "explicit modulo expansion," about which we shall have more to say later.

For merging two lists of $n$ elements, it has long been known that, in the serial case, $\Theta(n)$ steps are needed. In the balanced case, Valiant [V] showed that $O(\log \log n)$ steps are sufficient; Borodin and Hopcroft [BH2] showed that $\Omega(\log \log n)$ steps are necessary. In the highly parallel case, Häggkvist and Hell [HH3] showed that $\Theta\left(n^{1+1 /\left(2^{k}-1\right)}\right)$ comparisons are needed to merge in $k$ rounds.

For selecting an element of prescribed rank, it is desirable to distinguish between finding the minimum (or selecting an element of fixed rank), which seems to be easier than the general case, and finding the median, to which the general case can be reduced.

\footnotetext{
* Received by the editors February 18, 1986; accepted for publication (in revised form) January 21, 1987.

† IBM Almaden Research Laboratory K51-802, 650 Harry Road, San Jose, California 95120-6099.
} 
In the serial case, $\Theta(n)$ steps are needed (the bound of $O(n)$ steps for finding the median being due to Blum, Floyd, Pratt, Rivest and Tarjan [BFPRT]). In the balanced case, Valiant [V] showed that $\Omega(\log \log n)$ steps are necessary, and that $O(\log \log n)$ steps are sufficient for finding the minimum; Ajtai, Komlós, Steiger and Szemerédi [AKSS] showed that $O(\log \log n)$ steps are sufficient for finding the median.

In the highly parallel case, Häggkvist and Hell [HH1] showed that $\Omega\left(n^{1+1 /\left(2^{k}-1\right)}\right)$ comparisons are necessary for selection in $k$ rounds, and that $O\left(n^{1+1 /\left(2^{k}-1\right)}\right)$ comparisons are sufficient for finding the minimum in $k$ rounds. Alon, Azar and Vishkin [AAV] have shown that $\Omega\left(n^{1+1 /\left(2^{k}-1\right)}(\log n)^{2 /\left(2^{k}-1\right)}\right)$ comparisons are necessary for finding the median. In this paper we shall show that $O\left(n^{1+1 /\left(2^{k}-1\right)}(\log n)^{2-2 /\left(2^{k}-1\right)}\right)$ comparisons are sufficient for finding the median in $k$ rounds. This result is also "explicit modulo expansion."

The results that we have just described will be obtained by using a "probabilistic argument"; such an argument shows that a decision tree satisfying certain bounds exists without giving an algorithm (short of exhaustive search) for constructing one. Unlike most previous work on sorting in rounds, however, we shall use only one property of random graphs, namely, that with high probability a random graph is an "expanding graph." Considerable attention has been given to explicit constructions for expanding graphs, and it is not implausible that constructions for expanding graphs as good as those promised by probabilistic arguments will eventually be found. The results of this paper will then give explicit algorithms for sorting and selecting in rounds, achieving the bounds described above. (We have not defined formally what we mean by an "explicit construction." For the purposes of this paper we may take it to mean that the successive nodes in a decision tree may be computed in time polynomial in $n$.) Using what is presently known about the explicit construction of expanding graphs (see Lubotzky, Phillips and Sarnak [LPS]), we shall give explicit algorithms for sorting in $k$ rounds using $O\left(n^{1+2 /(k+1)}(\log n)^{2-4 /(k+1)}\right)$ comparisons, and selecting in $k$ rounds using $O\left(n^{1+2^{k-2} /\left(3^{k-1}-2^{k-2}\right)}(\log n)^{2-2^{k-1} /\left(3^{k-1}-2^{k-2}\right)}\right)$ comparisons.

The best previously known explicit algorithm for sorting in 2 rounds (due to Alon [A]) is $O\left(n^{7 / 4}\right)$, based on a projective geometry of dimension 4 . No better bound was known for selection. By applying Corollary 9 below with a projective geometry of dimension 3, we could give an explicit algorithm for sorting in 2 rounds using $O\left(n^{5 / 3} \log n\right)$ comparisons. By applying Proposition 6 with a projective geometry of dimension 2, we could give an explicit algorithm for selection in 2 rounds using $O\left(n^{3 / 2}(\log n)^{2}\right)$ comparisons. These bounds are only slightly weaker than those for $k=2$ obtained from the results of Lubotzky, Phillips and Sarnak, but projective geometries seem to give poor results for $k \geqq 3$. This difficulty may be traced to the fact that the dimension of a projective geometry must be an integer, and the results of Lubotzky, Phillips and Sarnak may be viewed as providing a surrogate for combinatorial geometries with continuously varying dimensions.

The approach taken in this paper is to assume that the number of rounds is fixed, and to minimize as far as possible the number of processors; we have only succeeded in doing this to within logarithmic factors. Another possible approach is to assume that the numbers of elements and processors are given, and to minimize as far as possible the number of rounds. For sorting, Alon, Azar and Vishkin [AAV] have shown that $\Theta(\log n / \log (1+p / n))$ rounds are necessary and sufficient with $p$ processors and $n$ elements. For merging, Kruskal $[\mathrm{K}]$ has shown that

$$
\Theta\left(n / p+\log \left(\frac{\log n}{\log (1+p / n)}\right)\right)
$$


comparisons are necessary and sufficient. It is natural to conjecture that a similar bound applies to finding the median, but it is not yet known whether $O(\log \log n)$ rounds suffice for $O(n / \log \log n)$ processors and $n$ elements.

\section{Statements and proofs of results.}

THEOREM 1. For every fixed integer $k \geqq 1$, there are algorithms (explicit modulo expansion) that select an element of prescribed rank from among $n$ elements in $k$ rounds using $O\left(n^{1+1 /\left(2^{k}-1\right)}(\log n)^{2-2 /\left(2^{k}-1\right)}\right)$ comparisons.

THEOREM 2. For every fixed integer $k \geqq 1$, there are algorithms (explicit modulo expansion) that sort $n$ elements in $k$ rounds using $O\left(n^{1+1 / k}(\log n)^{2-2 / k}\right)$ comparisons.

THEOREM 3. For every fixed integer $k \geqq 1$, there are explicitly constructed algorithms that select an element of prescribed rank from among $n$ elements in $k$ rounds using $O\left(n^{1+2^{k-2} /\left(3^{k-1}-2^{k-2}\right)}(\log n)^{2-2^{k-1} /\left(3^{k-1}-2^{k-2}\right)}\right)$ comparisons.

THEOREM 4. For every fixed integer $k \geqq 1$, there are explicitly constructed algorithms that sort $n$ elements in $k$ rounds using $\left(n^{1+2 /(k+1)}(\log n)^{2-4 /(k+1)}\right)$ comparisons.

The proofs of all these results will use expanding graphs. The following definition of expansion is best adapted to our purposes. Let $a$ be a nonnegative real number. An undirected graph is a-expanding if any two disjoint sets of vertices, each containing at least $a+1$ vertices, are joined by an edge. The key to our results is the following lemma, which is implicit in [AKSS].

LEMMA 5. In an a-expanding graph, for every set of vertices $X$ containing at least $5 a$ vertices, there exists a set of vertices $Y$ disjoint from $X$ and containing at most a vertices such that every set of vertices $Z$ disjoint from $X \cup Y$ and containing at most a vertices has at least $2|Z|$ neighbors in $X$, where $|Z|$ denotes the cardinality of $Z$.

Proof. Let $Y$ be a maximal (with respect to inclusion) set of vertices satisfying the following properties: (1) $Y$ is disjoint from $X$, (2) $Y$ contains at most $2 a$ vertices and (3) $Y$ has fewer than $2|Y|$ neighbors in $X$. Properties (2) and (3) imply that $Y$ has fewer than $4 a$ neighbors in $X$.

We claim first that $Y$ contains at most $a$ vertices. If not, then since the graph is $a$-expanding, all but at most $a$ of the vertices not in $Y$ would be neighbors of $Y$, so $X$ in particular would contain at least $5 a-a=4 a$ neighbors of $Y$, a contradiction. Finally, we observe that if $Z$ were a set violating the conclusion of the lemma, then $Y \cup Z$ would be a proper superset of $Y$ satisfying properties (1)-(3), contradicting the maximality of $Y$.

This lemma is used to prove the following propsition. We shall assume that our algorithms deal with $n$ distinct and totally ordered elements. If, after some comparisons have been performed, an element $v$ is known to be too large or too small to have rank $m$, we shall say that $v$ is excluded from rank $m$; otherwise we shall say that $v$ is a candidate for rank $m$. If $M$ is a set of ranks, we shall say that $v$ is excluded from $M$ if it is excluded from every rank in $M$; otherwise we shall say that it is a candidate for $M$.

PROPOSITION 6. If $n$ elements are compared according to the edges of an a-expanding graph, there will be $O(a \log n)$ candidates for any rank.

Proof. Suppose that the element of rank $m$ is being sought (where the minimum is the element of rank 1 and the maximum is the element of rank $n$ ). Let $b=$ $1+\left\lfloor\log _{2}(a+1)\right\rfloor=O(\log n)$. We shall show that, after the comparisons have been performed, all but at most $a(6 b+2)=O(a \log n)$ of the elements with rank greater than $m$ will be known to have rank greater than $m$. By the dual argument, all but at most $a(6 b+2)$ of the elements with rank less than $m$ will be known to have rank less than $m$, completing the proof. 
If $m>n-a(6 b+1)$, then there are fewer than $a(6 b+1)$ elements with rank greater than $m$, and there is nothing to prove. Otherwise, classify the elements into three classes as follows. Let $G_{-}$contain the smallest $m+a$ elements, let $G_{0}$ contain the next $a(6 b+1)$ elements, and let $G_{+}$contain the remaining elements. Since $G_{-} \cup G_{0}$ contains just $a(6 b+1)$ elements with rank greater than $m$, it will suffice to show that $G_{+}$contains at most $a$ such elements.

Further classify the elements of $G_{0}$ into $b$ subclasses $G_{1}, \cdots, G_{b}$, with $G_{1}$ containing the smallest $6 a$ elements of $G_{0}, \cdots$, and with $G_{b}$ containing the largest $6 a$ elements of $G_{0}$. Finally, classify the elments in each of the classes $G_{1}, \cdots, G_{b}$ as "good" or "bad" as follows. All of the elements of $G_{1}$ are good. Suppose that, for some $1 \leqq c \leqq$ $b-1$, the elements of $G_{c}$ have been classified and that there are at least $5 a$ good elements in $G_{c}$. To classify the elements of $G_{c+1}$, apply Lemma 5 with $X$ as the set of good elements in $G_{c}$. Classify as bad those elements of $G_{c+1}$ that belong to the resulting set $Y$, of which there are at most $a$; classify as good the remaining elements of $G_{c+1}$, of which there are at least $6 a-a=5 a$. By Lemma 5 , this classification has the following property: any set $Z$ of $z \leqq a$ good elements in $G_{c+1}$ has at least $2 z$ good neighbors in $G_{c}$.

If an element is known to be larger than at least $a+1$ good elements in $G_{1}$, then by comparisons according to an $a$-expanding graph, it is also known to be greater than all but at most $a$ elements in $G_{-}$, and thus is known to have rank greater than $m$. We shall show by induction on $c$ that if, for some $0 \leqq c \leqq b-1$, an element is known to be larger than at least $(a+1) / 2^{c}$ good elements in $G_{c+1}$, then it is known to have rank greater than $m$. The basis, $c=0$, is the foregoing observation. Suppose that $c \geqq 1$ and that the element $v$ is known to be larger than at least $(a+1) / 2^{c}$ good elements in $G_{c+1}$. These good elements in $G_{c+1}$ have at least $2(a+1) / 2^{c}=(a+1) / 2^{c-1}$ good neighbors in $G_{c}$. By transitivity, $v$ is known to be larger than these elements in $G_{c}$, and thus by inductive hypothesis $v$ is known to have rank greater than $m$. This completes the inductive step. Taking $c=b-1$, we see that if an element is known to be larger than at least one good element in $G_{b}$, then it is known to have rank greater than $m$.

The set $G_{b}$ contains at least $5 a \geqq a+1$ good elements. Thus, by comparisons according to the edges of an $a$-expanding graph, all but at most $a$ elements in $G_{+}$are known to be larger than at least one good element in $G_{b}$. Thus all but at most $a$ elements in $G_{+}$are known to have rank greater than $m$.

To prove Theorem 1, we shall use this proposition together with the following lemma.

LEMMA 7. For all $1 \leqq a \leqq n$ with $n$ sufficiently large, there is an a-expanding graph with $n$ vertices and $O\left(\left(n^{2} \log n\right) / a\right)$ edges.

Proof. If $a \leqq 2 \ln n$, the bound $O\left(n^{2}\right)$ is trivial, so suppose $a \geqq 2 \ln n$. Let $G$ be a random graph on $n$ vertices in which each edge is independently present with probability $p$ and absent with probability $1-p$, where $p=(2 \ln n) / a$. We shall show that (1) the probability that $G$ contains two disjoint sets of $a+1$ vertices not joined by an edge is at most $1 / n^{2}$, and (2) the probability that $G$ contains more than $2\left(n^{2} \ln n\right) / a$ edges is at most $1 /(n \ln n)$. Since $1 / n^{2}+1 /(n \ln n)<1$ when $n \geqq 2$, the lemma will follow.

To prove (1), we observe that there are

$$
\left(\begin{array}{c}
n \\
a+1, a+1
\end{array}\right)<n^{2(a+1)}
$$

ways to choose two disjoint sets of $a+1$ vertices, and that for each choice, the probability that no edge joins them is

$$
(1-p)^{(a+1)^{2}}<e^{-p(a+1)^{2}}
$$


Thus, the probability of the event in question is at most

$$
n^{2(a+1)} e^{-p(a+1)^{2}}=\left(n^{2} e^{-p(a+1)}\right)^{a+1}=e^{-p(a+1)}<1 / n^{2} .
$$

To prove (2), we observe that the mean and variance of the number of edges in $G$ are $\left(\begin{array}{c}n \\ 2\end{array}\right) p<\left(n^{2} \ln n\right) / a$ and $\left(\begin{array}{c}n \\ 2\end{array}\right) p(1-p)<\left(n^{2} \ln n\right) / a$, respectively. Thus, by Chebyshev's inequality, the probability that $G$ contains more than $2\left(n^{2} \ln n\right) / a$ edges is at most $a /\left(n^{2} \ln n\right)<1 /(n \ln n)$.

Proof of Theorem 1. We proceed by induction on $k$. If $k=1$, the bound $O\left(n^{2}\right)$ is trivial. If $k \geqq 2$, let

$$
a=n^{1-1 /\left(2^{k}-1\right)} /(\ln n)^{1-2 /\left(2^{k}-1\right)} .
$$

Compare the elements according to the edges of the graph supplied by Lemma 7. By Proposition 6, at most

$$
t=O(a \log n)=O\left(n^{1-1 /\left(2^{k}-1\right)}(\log n)^{2 /\left(2^{k}-1\right)}\right)
$$

elements will remain as candidates. By inductive hypothesis, the element sought can be selected from these candidates in $k-1$ rounds using

$$
O\left(t^{1+1 /\left(2^{k-1}-1\right)}(\log t)^{2-2 /\left(2^{k-1}-1\right)}\right)=O\left(n^{1+1 /\left(2^{k}-1\right)}(\log n)^{2-2 /\left(2^{k}-1\right)}\right)
$$

further comparisons.

To prove Theorem 3, we shall use Proposition 6 together with explicitly constructed expanding graphs provided by the following lemma.

LEMMA 8. Let $p$ and $q$ be distinct primes congruent to 1 modulo 4 , with $p<q$. Then there is an explicitly constructed $\left(2(q+1) /(p+1)^{1 / 2}\right)$-expanding graph with $q+1$ vertices and $(p+1)(q+1) / 2$ edges.

Proof. Lubotzky, Phillips and Sarnak [LPS] have shown that if $p$ and $q$ satisfy the hypotheses of the lemma, there is an explicitly constructed graph $G$ with $q+1$ vertices and $p+1$ edges meeting every vertex such that (1) the largest eigenvalue $p+1$ of the adjacency matrix $M$ of $G$ has multiplicity 1 and (2) all other eigenvalues of $M$ have magnitude at most $2 p^{1 / 2}$. We shall show that $G$ is $\left(2(q+1) /(p+1)^{1 / 2}\right)$-expanding.

Suppose that $A$ and $B$ are disjoint sets of $m$ elements that are not joined by an edge in $G$. We shall show that $m \leqq 2(q+1) /(p+1)^{1 / 2}$.

Let $e$ be the function that assigns the value 1 to each vertex of $G$. Then $\langle e, e\rangle=q+1$ and $e$ is the unique eigenvector of $M^{T} M$ (which equals $M^{2}$, since $M$ is symmetric) corresponding to the eigenvalue $(p+1)^{2}$.

Let $f$ be the function that assigns the value 1 to vertices in $A$ and the value 0 to all other vertices. Then $\langle f, f\rangle=m$. Let the functions $g=e\langle e, f\rangle / n$ be the part of $f$ lying parallel to $e$ and let $h=f-g$ be the part of $f$ lying perpendicular to $e$. We shall estimate $\left\langle f, M^{T} M f\right\rangle=\langle M f, M f\rangle$ in two ways.

First, $\left\langle f, M^{T} M f\right\rangle=\left\langle g, M^{T} M g\right\rangle+\left\langle h, M^{T} M h\right\rangle$. Thus

$$
\begin{aligned}
\left\langle f, M^{T} M f\right\rangle & \leqq(p+1)^{2}\langle g, g\rangle+4 p\langle h, h\rangle \\
& =\frac{(p+1)^{2} m^{2}}{n}+\frac{4 p m(n-m)}{n},
\end{aligned}
$$

since all eigenvalues of $M^{T} M$ other than $(p+1)^{2}$ have magnitude at most $4 p$.

Second, let $d$ be the function that assigns the value 0 to vertices in $B$ and 1 to all other vertices. By Cauchy's inequality, $\langle M f, M f\rangle \geqq\langle M f, d\rangle^{2} /\langle d, d\rangle$. Now the function $M f$ assigns to each vertex the number of neighbors it has in $A$. Since no vertex of $B$ 
has a neighbor in $A, M f$ vanishes on all vertices for which $d$ vanishes. Thus $\langle M f, d\rangle=$ $\langle M f, e\rangle=\left\langle f, M^{T} e\right\rangle=(p+1) m$ and, of course, $\langle d, d\rangle=n-m$. Thus we have

$$
\langle M f, M f\rangle \geqq \frac{(p+1)^{2} m^{2}}{n-m} \text {. }
$$

Combining this inequality with that of the preceding paragraph yields $m \leqq$ $2(q+1) /(p+1)^{1 / 2}$ and completes the proof.

Proposition 9. For all $1 \leqq a \leqq n$ with $n / a$ sufficiently large, there is an explicitly constructed a-expanding graph with $n$ vertices and $O\left(n^{3} / a^{2}\right)$ edges.

Proof. If $a \leqq 8 n^{1 / 2}$, the bound $O\left(n^{2}\right)$ is trivial, so suppose $4(4 n / a)^{2} \leqq n$. Take $x=(4 n / a)^{2}-1$ and $y=n-1$. By the prime number theorem for arithmetic progressions (see Davenport [D], for example), the number $\pi_{1,4}(x)$ of primes congruent to 1 modulo 4 and at most $x$ satisfies $\pi_{1,4}(x) \sim x /(2 \ln x)$. It follows that $\pi_{1,4}(2 x)-\pi_{1,4}(x) \sim x / 2 \ln x$. Thus for all sufficiently large $n / a$ there is a prime $p$ congruent to 1 modulo 4 and satisfying $x \leqq p \leqq 2 x$ and therefore $(4 n / a)^{2} \leqq p+1 \leqq 2(4 n / a)^{2}$. Similarly, there is a prime $q$ congruent to 1 modulo 4 and satisfying $y \leqq q \leqq 2 y$ and therefore $n \leqq q+1 \leqq 2 n$. Furthermore, since $2(4 n / a)^{2}<n$, we have $p<q$. Applying Lemma 8 , we obtain a $\left(2(q+1) /(p+1)^{1 / 2}\right)$-expanding graph with $q+1$ vertices and $(p+1)(q+1) / 2=$ $O\left(n^{3} / a^{2}\right)$ edges. Since $2(q+1) /(p+1)^{1 / 2} \leqq 4 n /(p+1)^{1 / 2} \leqq a$, this graph is $a$-expanding. By identifying $q+1-n$ disjoint pairs of vertices to form single vertices we obtain a graph with $n$ vertices, the same number of edges and the same expanding property.

Proof of Theorem 3. We proceed as in the proof of Theorem 1, but let

$$
a=n^{1-2^{k-3} /\left(3^{k-1}-2^{k-2}\right)} /(\ln n)^{1-2^{k-2} /\left(3^{k-1}-2^{k-2}\right)}
$$

and use Proposition 9.

To prove Theorems 2 and 4, we shall use the following corollary of Proposition 6.

COROLLARY 10. If $n$ elements are compared according to the edges of an a-expanding graph, then after the comparisons have been performed we may construct $O(n /(a \log n))$ sets, each containing $O(a \log n)$ elements, such that the relationship between any pair of elements is known unless they both belong to a common set.

Proof. Partition the ranks into $O(n /(a \log n))$ contiguous intervals, each containing at most $O(a \log n)$ successive ranks. Let $[i, j]$ be such an interval. By Proposition 6 , all but at most $O(a \log n)$ of the elements with rank less than $i$ are known to have rank less than $i$, and all but at most $O(a \log n)$ of the elements with rank greater than $j$ are known to have rank greater than $j$. Since at most $O(a \log n)$ elements have ranks in $[i, j]$, at most $O(a \log n)$ elements are candidates for the interval $[i, j]$. The sets of elements that we construct will be the sets of candidates for the intervals.

If an element is not a candidate for an interval, then either it is known to be too large to have its rank in that interval or it is known to be too small to have its rank in that interval. It follows that the family of intervals for which an element is a candidate is contiguous.

Consider any pair $v$ and $w$ of elements. If $v$ and $w$ do not both belong to a common set, then the family $A$ of intervals for which $v$ is a candidate is disjoint from the family $B$ of intervals for which $w$ is a candidate. Since these families are each contiguous, either all intervals of $A$ are less than all intervals of $B$ (in which case $v$ must be less than $w$ ) or all intervals of $A$ are greater than all intervals of $B$ (in which case $v$ must be greater than $w$ ).

Proof of Theorem 2. We proceed by induction on $k$. If $k=1$, the bound $O\left(n^{2}\right)$ is trivial. If $k \geqq 2$, let

$$
a=n^{1-1 / k} /(\ln n)^{1-2 / k}
$$


Compare the elements according to the edges of the graph supplied by Lemma 7 . By Corollary 10, we may construct

$$
s=O(n /(a \log n))=O\left(n^{1 / k}(\log n)^{-2 / k}\right)
$$

sets each containing

$$
t=O(a \log n)=O\left(n^{1-1 / k}(\log n)^{2 / k}\right)
$$

elements and it will suffice to sort each of these sets. By inductive hypothesis, these sets can be sorted in $k-1$ rounds using

$$
O\left(s t^{1+1 /(k-1)}(\log t)^{2-2 /(k-1)}\right)=O\left(n^{1+1 / k}(\log n)^{2-2 / k}\right)
$$

further comparisons.

Proof of Theorem 4. We proceed as in the proof of Theorem 2, but let

$$
a=n^{1-1 /(k+1)} /(\ln n)^{1-2 /(k+1)}
$$

and use Proposition 9.

\section{REFERENCES}

[AKS] M. Ajtai, J. Komlós AND E. Szemerédi, Sorting in $c \log n$ steps, Combinatorica, 3 (1983), pp. 1-19.

[AKSS] M. AJtai, J. Komlós, W. L. Steiger and E. Szemerédi, Deterministic selection in $O(\log$ $\log n$ ) parallel time, ACM Symp. on Theory of Computing, 18 (1986), pp. 188-195.

[A] N. ALON, Eigenvalues, geometric expanders, sorting in rounds, and Ramsey theory, Combinatorica, 6 (1986), pp. 207-219.

[AAV] N. Alon, Y. AzAR AND U. Vishkin, Tight complexity bounds for parallel comparison sorting, IEEE Symp. on Foundations of Computer Science, 27 (1986), pp. 502-510.

[BFPRT] M. Blum, R. Floyd, V. Pratt, R. Rivest AND R. TARJAN, Time bounds for selection, J. Comput. System Sci., 7 (1973), pp. 448-461.

[BH1] B. Bollobás AND P. Hell, Sorting and graphs, in Graphs and Order, I. Rival, ed., Reidel, Boston, 1985, pp. 169-184.

[BT] B. Bollobás And A. Thomason, Parallel sorting, Discrete Appl. Math., 6 (1983), pp. 1-11.

[BH2] A. BORODIN AND J. E. HOPCROFT, Routing, merging, and sorting on parallel models of computation, J. Comput. System Sci., 30 (1985), pp. 130-145.

[D] H. DAvenport, Multiplicative Number Theory, 2nd edition, Springer, New York, 1980.

[HH1] R. HÄGGKVIST AND P. HELl, Graphs and parallel comparison algorithms, Congr. Numer., 29 (1980), pp. 497-509.

[HH2] Parallel sorting with constant time for comparisons, this Journal, 10 (1981), pp. 465-472.

[HH3] R. HÄGGKVIST AND P. Hell, Sorting and merging in rounds, SIAM J. Algebraic Discrete Methods, 3 (1982), pp. 465-473.

[K] C. P. KRUSKAL, Searching, merging and sorting in parallel computation, IEEE Trans. Comput., 32 (1983), pp. 942-946.

[LPS] A. LubOtZky, R. Phillips ANd P. SARNAK, Explicit expanders and the Ramanujan conjectures, ACM Symp. on Theory of Computing, 18 (1986), pp. 240-246.

[V] L. G. VAliant, Parallelism in comparison problems, this Journal, 4 (1975), pp. 348-355. 